\title{
Joint Spectrum Allocation and Power Control for Multihop Cognitive Radio Networks
}

\author{
Wei Wang, Member, IEEE, Kang G. Shin, Fellow, IEEE, and Wenbo Wang, Member, IEEE
}

\begin{abstract}
We propose a new framework of joint spectrum allocation and power control to utilize open spectrum bands in cognitive radio networks (CRNs) by considering both interference temperature constraints and spectrum dynamics. We first address a simpler problem for the case of a single flow. A TDM-based power-control strategy is adopted to achieve maximum end-to-end throughput by choosing an appropriate multihop route and spectrum combination for each single flow. Then, the simpler solution is extended to the multiflow case in which interflow interference and cumulative interference temperature must be considered. Considering the overhead of switching route and spectrum, the optimal waiting time before making a switch is derived. Our in-depth simulation study has shown that the proposed algorithms utilize spectrum more efficiently than other existing algorithms.
\end{abstract}

Index Terms-Cognitive radio networks (CRNs), multiple-hop flows, spectrum allocation, power control.

\section{INTRODUCTION}

$\mathrm{T}$ HE problem of severely unbalanced, and hence inefficient, spectrum utilization [1] has been attracting considerable attention from academia and industry as the target for remedying the anticipated spectrum shortage in accommodating rapidly growing wireless applications and user population. Static spectrum allocation regulation is known to be the main culprit of inefficient spectrum utilization because a licensed spectrum band can be used only by its primary/licensed users. Unlicensed users are not allowed to use the spectrum band even when it is left idle by the primary users.

To mitigate this problem, the FCC is considering the fixed spectrum license regulation to be relaxed, thus allowing unlicensed/secondary users (SUs) to access idle spectrum bands dynamically, i.e., secondary users can utilize the spectrum as long as they do not interfere with the primary users. Cognitive radios [2] and their internetworking are an important means of realizing this spectrum utilization improvement. The authors of [3], [4] discussed the main characteristics of CRNs, including signal processing, spectrum utilization, and adaptive procedures. Note, however, that most previous research on cognitive radios focused on single-hop communications.

For multihop wireless networks, we must simultaneously consider spectrum allocation and power control, which is a very important but difficult problem. Previous related work

- W. Wang is with the Institute of Information and Communication Engineering, Key Laboratory of Integrated Information Network Technology, Zhejiang University, Zheda Rd. 38, Hangzhou 310027, P.R. China. E-mail:wangw@zju.edu.cn.

- K.G. Shin is with the Real-Time Computing Laboratory, Department of Electrical Engineering and Computer Science, University of Michigan, 2260 Hayward St., Ann Arbor, MI 48109.E-mail: kgshin@umich.edu.

- W. Wang is with the Wireless Signal Processing and Network Lab, Beijing University of Posts and Telecommunications, PO Box 93, Xitucheng Rd. 10, Beijing 100876, P.R. China. E-mail: wbwang@bupt.edu.cn.

Manuscript received 11 Aug. 2008; revised 3 July 2010; accepted 12 Aug. 2010; published online 25 Oct. 2010.

For information on obtaining reprints of this article, please send e-mail to: tmc@computer.org, and reference IEEECS Log Number TMC-2008-08-0318. Digital Object Identifier no. 10.1109/TMC.2010.207. attempted to minimize power consumption [5], [6]. The optimal power-control scheme in [5] determines the power consumption per unit of data transmitted, and then, iteratively allocates the required data rate to different routes. In [6], a conflict-free spectrum allocation is deployed first, and joint route selection and power control is then designed using the spectrum allocation. To maximize throughput, the joint power and link-scheduling problem is formulated as a mixed integer linear programming problem in [7]. The objective function used is not the end-to-end (E2E) throughput but the sum of all the links' throughput. In [8], Time Division Multiplexing (TDM) is used for link scheduling without considering transmit power.

Joint spectrum allocation and power control in multihop CRNs introduces several new challenges. First, the most important issue in CRNs is to protect primary users' communications. Choosing an idle spectrum, ${ }^{1}$ allocating the spectrum to the links far away from the primary receivers using this spectrum, and applying power control to keep the interference to the primary users below the required limit must all be considered. Second, the spectrum condition changes according to the primary users' activities, and hence, we need a strategy that adapts to the spectrum dynamics without incurring too much overhead in switching spectrum and route. An appropriate scheme of switching spectrum and route is, therefore, necessary to make a trade-off between the spectrum utilization and the switching overhead.

There have been only a few publications on routing and resource allocation for multihop CRNs. The authors of [9] described decoupled and collaborative interactions between route selection and spectrum management. In [10], a lowcomplexity algorithm is proposed to obtain a Pareto optimal solution for selecting a minimum-delay route. In [11], an ondemand routing protocol is proposed, also to minimize the delay, but with different delay definitions from those in

1. The term "spectrum" is used to represent the channels in a radio spectrum, and hence, we will use "spectrum" and "channel(s)" interchangeably. 
[10]. In [12] and [13], a graph-coloring model is adopted for interference-free channel allocation. In the above-mentioned multihop CRNs, the secondary users are allowed to use the licensed spectrum only when it is not being used by the primary users, and power control was not considered at all. In practice, however, with proper power control, the secondary users can transmit data using low power that causes only acceptably low interference to the primary users' communications. This way, the spectrum efficiency can be improved further.

In [14], joint channel and power allocation is investigated for CRNs. The authors of [15] focus on spectrum and power allocation in infrastructure-based CRNs. They consider the intercell interference for sharing the spectrum. In [16], interference temperature is adopted to restrict the interference to primary users. In [17], the spectrum sharing and routing problem is considered under the assumption that the set of available channels to each secondary user is static. In [18] and [19], centralized and distributed schemes are proposed for CRNs, respectively. Both of them only consider different available channels at each node. In [20], the end-to-end throughput optimization problem is divided into two subproblems and is optimized using the Lagrangian duality. However, these approaches do not consider power control together with spectrum allocation for optimizing end-to-end throughput in a CRN. Moreover, they do not consider the dynamics of the available spectrum along with the resource allocation under an interference temperature model.

By contrast, in this paper, we address joint spectrum allocation and power control for secondary users which coexist with primary users on the same spectrum. We consider both the dynamic spectrum model [12] and the interference temperature model [16]. In the former, the primary users may not always use the spectrum, and hence, the secondary users can opportunistically utilize the spectrum when it is not being used by the primary users. In the latter, both primary and secondary users can coexist on the same spectrum. The secondary users' interference to the primary receivers should not exceed the interference temperature limit. In most previous research, only one of these two models is considered for simplicity, but we consider both simultaneously; hence, addressing more general cases and improving the spectrum efficiency.

The main contributions of this paper are as follows:

- We present a new framework for characterizing the interplay among spectrum allocation and power control.

- We develop a TDM-based power-control scheme. An optimal time-partitioning strategy is derived to achieve the maximum end-to-end throughput, which is then used to evaluate the performance of each candidate route-spectrum combination.

- We develop a distributed spectrum allocation and power-control strategy for each flow. Nodes cooperate to deal with the interflow interferences and the cumulative interference temperature to enable the coexistence of multiple flows.

- The optimal waiting time before switching route and spectrum is derived by making a trade-off between
TABLE 1

List of the Basic Notations for the Network Model

\begin{tabular}{ll}
\hline Symbol & \multicolumn{1}{c}{ Meaning } \\
\hline$p_{l}$ & transmit power of link $l$ \\
$p_{l}^{\max }$ & peak transmit power of link $l$ \\
$\gamma_{l}$ & signal-to-interference ratio of link $l$ \\
$g_{l j}$ & path gain from the transmitter of link $l$ to \\
the receiver of link $j$
\end{tabular}

the spectrum efficiency and the overhead of switching route and spectrum.

The rest of the paper is organized as follows: Section 2 describes the multihop network and spectrum models we will consider. Section 3 presents a new framework of joint spectrum allocation and power control in CRNs. Sections 4-6 describe power control, interflow coordination, and routespectrum switch, respectively. Finally, the paper concludes with Section 7.

\section{System Models}

\subsection{Multihop Network Model}

We consider a multihop CRN that consists of a set $\mathcal{N}$ of nodes and a set $\mathcal{L}$ of links. There is a set $\mathcal{K}$ of $K$ available spectrum bands. Each node is equipped with two radio interfaces, one for transmitting data and the other for receiving data, so the node can transmit and receive data at the same time. Like most typical wireless transceivers, the node can only work on one channel at a time for each interface. Table 1 lists the notations of the basic parameters in the network model.

Let $\gamma_{l}$ be the signal-to-interference ratio (SIR) on link $l$ :

$$
\gamma_{l}=\frac{p_{l} g_{l l}}{\sigma^{2}+\sum_{j \in \mathcal{L}(k(l)), j \neq l} p_{j} g_{j l}},
$$

where $k(l)$ is the spectrum on link $l, k(l) \in \mathcal{K}, \mathcal{L}(k)$ is the link set on spectrum $k$, and $\sigma^{2}$ includes not only the thermal noise, but also the interference from the primary users. According to Shannon's capacity formula, the throughput over link $l$ can be modeled as a function of SIR:

$$
R_{l}=W \log \left(1+\gamma_{l}\right),
$$

where $W$ is the bandwidth of a spectrum band.

Each node has a peak transmit power limit. The transmit power on each link should be kept below the peak power of its transmitter because of the equipment capability, i.e.,

$$
p_{l} \leq p_{l}^{\max } .
$$


TABLE 2

List of the Basic Symbols for the Spectrum-Activity Model

\begin{tabular}{ll}
\hline Symbol & Meaning \\
\hline$T_{k}^{O N}$ & duration of spectrum $k^{\prime}$ s ON state \\
$T_{k}^{O F F}$ & duration of spectrum $k^{\prime}$ s OFF state \\
$f_{k}^{O N}(t)$ & probability density function of $T_{k}^{O N}$ \\
$f_{k}^{O F F}(t)$ & probability density function of $T_{k}^{O F F}$ \\
$T_{S, k}^{O N}$ & waiting time before switching spectrum $k$ from \\
$T_{S, k}^{O F F}$ & OFF to ON \\
$t_{S}$ & waiting time before switching spectrum $k$ from \\
& ON to OFF \\
\hline
\end{tabular}

The data of a set $\mathcal{F}$ of flows from different source nodes are transmitted to their corresponding receivers/destinations in a multihop CRN. Suppose each flow is routed from its source to its destination via a single path.

There is a set $\mathcal{M}$ of $M$ primary receivers in the network. Primary users have priority over secondary users in accessing the spectrum; hence, restricting the secondary users' spectrum usage as we describe next.

\subsection{Spectrum-Activity Model}

Two types of models have been proposed so far for the primary users' spectrum usage activities: dynamic spectrum model [12] and interference temperature model [16].

According to the primary users' spectrum usage pattern based on the experimental results in [21] and [22], the spectrum usage can be modeled as an ON-OFF process: ON (OFF) state represents when the spectrum is occupied (unoccupied) by primary users. In order to protect the primary users' communications, the interference caused by secondary users must be kept below the interference temperature limit at the primary receivers. That is, the primary users' Quality-of-Service (QoS) is acceptable if the secondary users' interference is kept below a given interference temperature limit:

$$
\sum_{l \in \mathcal{L}(k)} p_{l} h_{l m} \leq Q_{m k}^{\max }, \quad \forall m \in \mathcal{M} .
$$

Here, we assume that the interference temperature limits are known to the secondary users (cognitive radios) via some methods, such as use of dedicated control channels or backbone networks. In OFF state, the spectrum can be used by secondary users without considering their interference to primary users. It is assumed that the secondary users can obtain the perfect information on a primary user's return.

The spectrum dynamics can be modeled as a semiMarkov process as in [23]. The lengths of ON and OFF periods are independent and identically distributed (i.i.d.). Table 2 lists the symbols used in the spectrum-activity model. These parameters of spectrum activities can be estimated by secondary users, e.g., using maximum likelihood (ML) estimators. The authors of [23] gave details of the ML method for estimating the distribution parameters, but it is beyond the scope of this paper.

\subsection{Strategy-Switch Model}

As mentioned earlier, the status of spectrum changes according to the primary users' activities on the spectrum. To improve spectrum utilization, the SUs need to adapt their spectrum-use strategies. It takes the SUs a nonzero amount of time, say $t_{S}$, to switch their route and spectrum. During this time, no data transmission is allowed, thus incurring a switching overhead. Considering this overhead, it is not always efficient to make a switch immediately upon change of the spectrum status $(\mathrm{ON} \rightarrow \mathrm{OFF}$ or $\mathrm{OFF} \rightarrow \mathrm{ON})$ because frequent route and spectrum switches will incur large overheads which may offset the gain achieved from the switchings. Therefore, for spectrum $k$, a route and spectrum switch is made only after waiting a certain amount of time, denoted by $T_{S, k}^{O N}$ and $T_{S, k}^{O F F}$ as defined in Table 2. Fig. 1 presents a state-transition diagram showing the transition conditions between the strategies associated with ON/OFF states.

During the waiting period (WP), a different strategy is used to protect primary users when the spectrum band makes an $\mathrm{ON} \rightarrow \mathrm{OFF}$ or $\mathrm{OFF} \rightarrow \mathrm{ON}$ transition as follows:

- $\mathrm{OFF} \rightarrow \mathrm{ON}$ transition: SUs do not transmit/receive any information to protect primary users, as WP1 and WP3 in Fig. 2.

- $\mathrm{ON} \rightarrow \mathrm{OFF}$ transition: SUs continue transmission/ receiption using the current strategy, as WP2 and WP4 in Fig. 2.

When there are $K(>2)$ spectrum bands, if the waiting time on one of the bands reaches the threshold of a corresponding switch, a route and spectrum switch will be made. Fig. 2 provides an example switch after WP3. When the waiting time on spectrum 3 reaches $T_{S, 3}^{O N}$, the strategy is switched.

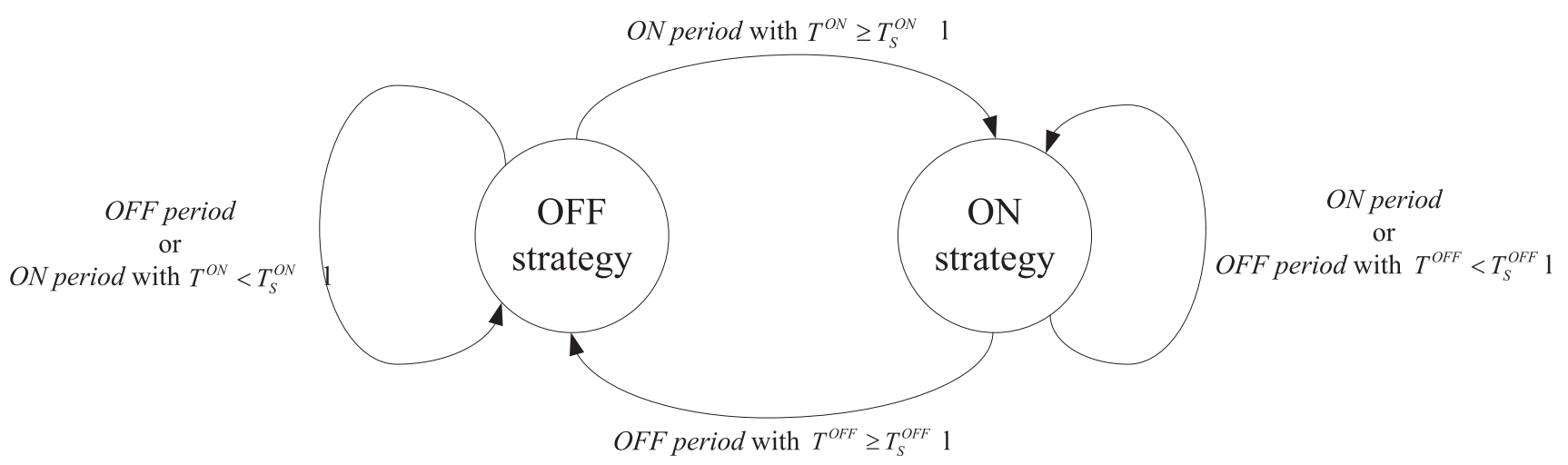

Fig. 1. Strategy-switch conditions with alternate spectrum ON/OFF status. 


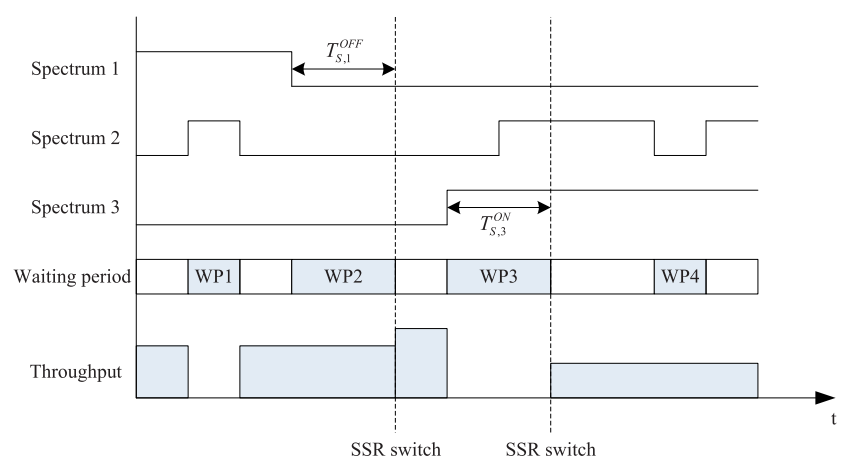

Fig. 2. An example of spectrum utilization of a flow.

\section{Open Spectrum Utilization}

Our problem is to enable the flows to utilize the spectrum efficiently, which can be stated formally as

$$
\max \sum_{f \in \mathcal{F}} U^{f}\left(R^{f}\right)
$$

subject to the constraints of peak transmit power (3) and interference temperature limit (4). In this problem formulation, $U^{f}($.$) is the utility function of data rate R^{f}$ for flow $f$, which could be $\log R^{f}$ for proportional fairness, or $a_{f} R^{f}$ for maximum weighted throughput. $R^{f}$ is the end-to-end throughput of flow $f$ which is dictated by the link with the minimum throughput as

$$
R^{f}=\min _{l \in \mathcal{L}(f)} R_{l},
$$

where $\mathcal{L}(f)$ is the set of links through which flow $f$ runs.

For efficient spectrum utilization, we propose a framework of joint spectrum allocation and power control for secondary users. In this framework, three subproblems are solved iteratively at different levels as follows:

\subsection{Optimization for a Single Flow}

This subproblem is to maximize $R^{f}$ for each flow $f$. Route and spectrum are jointly considered by selecting a strategy from all possible route-spectrum combinations based on the throughput to be achieved. We call a route-spectrum combination a spectrum-specified route (SSR). For each candidate SSR of a single flow, we estimate its end-to-end throughput. The throughput estimation is based on TDMbased power control, as we will describe in Section 4. We select the SSR with the maximum E2E throughput. Even though this subproblem is only for maximizing the E2E throughput of a single flow, it has to consider more complex factors, such as the interference/conflict between flows, allowable interference to primary users, and the inefficiency caused by spectrum dynamics. Therefore, we need to consider the following two subproblems together.

\subsection{Optimization for Multiple Flows}

The objective of this subproblem is to maximize $\sum_{f \in \mathcal{F}} U^{f}\left(R^{f}\right)$ during a period in which the states of channels do not change. According to our problem formulation, the binding constraints related to multiple flows are the interference in (1) and the interference temperature constraints (4). When the data of several flows are transmitted simultaneously in a multihop CR network, all flows will decide on their spectrum allocation and power control in a distributed manner. In order to achieve good performance and protect the primary users, these concurrent flows need to cooperate when selecting an SSR and determining the corresponding power level. This cooperation among multiple flows will be addressed in Section 5. The cooperation between flows will set some parameters for the flow-based distributed scheme, such as the successful transmission probability on a link with collisions considered, and a flow's allowable interference to primary users.

\subsection{Optimization in a Dynamic Spectrum Environment}

This subproblem is to determine the optimal waiting time before making an SSR switch. If the PUs' spectrum-usage pattern changes, the SSR should also be changed. If SSR switch immediately upon change of the spectrum status, each CR or SU may achieve better spectrum utilization, but frequent SSR switches will incur large overhead. Therefore, an SSR switch should wait for a certain amount of time, not immediately upon change of the spectrum status. In Section 6, we will make a trade-off between the spectrum efficiency and the spectrum switch overhead. Different values of the waiting time will yield different throughput loss of links, affecting the strategy decision when each flow optimizes its own objective.

\section{TDM-Based Power Control for SSR}

We first consider a simple case: the network with only a single flow. When selecting an SSR, we need to know the underlying power-control model in order to estimate the end-to-end throughput of each candidate SSR.

\subsection{Power-Control Model}

We propose a new parameter called the effective time portion and then divide the problem into several subproblems for each spectrum.

For a given SSR, instead of (6), the long-term mean endto-end throughput of flow $f$ is dictated by the bottleneck link on this path as

$$
R^{f}=\min _{l \in \mathcal{L}(f)}\left(\theta_{k(l)} \mathbb{E}\left[R_{l}\right]\right),
$$

where $\mathbb{E}[$.$] is the expectation. In (7), the effective time$ portion $\theta_{k}$ is introduced into the formulation of $R^{f}$ for spectrum $k$. If the strategy is determined with spectrum $k$ in ON state, then $\theta_{k}=1$ because the SU does not change his strategy during the wait before making an SSR switch. If spectrum $k$ is in OFF state, in order to protect PUs, the SU does not transmit/receive data during the wait after the spectrum changes to $\mathrm{ON}$ state, so a portion of time is used to avoid the interference to PUs, e.g., WP1 and WP3 in Fig. 2. The effective time portion for the spectrum in OFF state is

$$
\theta_{k}=\frac{\int_{0}^{\infty} t f_{k}^{O F F}(t) d t}{\int_{0}^{T_{S, k}^{O N}} t f_{k}^{O N}(t) d t+\int_{0}^{\infty} t f_{k}^{O F F}(t) d t} .
$$

Suppose there is no power leakage between the neighboring spectrum bands, meaning that the links on different spectrum bands do not interfere with each other. So, we only need to consider how to control the transmit 
power over the links on the same spectrum. Let $R_{k}^{f}$ denote the minimum link throughput on spectrum $k$ on the path of flow $f$, then

$$
R_{k}^{f}=\min _{l \in \mathcal{L}(f), k(l)=k}\left(\mathbb{E}\left[R_{l}\right]\right) .
$$

Based on the minimum throughput for each part of links, the E2E throughput of a flow, which is restricted by the minimum link throughput of the flow's path, is dictated by the minimum of $R_{k}^{f}$ over the spectrum bands $k \in \mathcal{K}(f)$, where $\mathcal{K}(f)$ is the set of spectrum bands used by flow $f$ :

$$
R^{f}=\min _{k \in \mathcal{K}(f)}\left(\theta_{k} R_{k}^{f}\right)
$$

This way, the throughput optimization problem of flow $f$ is decomposed into multiple subproblems $\max R_{k}^{f}$ for each spectrum $k$.

\subsection{TDM-Based Suboptimal Power Control}

Each link has its own transmitter and receiver, and transmissions over the links on the same spectrum may interfere with each other. This can be modeled as the interference channel model from an information-theoretic perspective [24], [25], which is still an open problem, even without interference temperature constraints. The optimal strategy for the capacity of interference channels depends on the interference coefficients, which correspond to the ratio of the path gains of interference signals to those of useful signals in the system model. From [24], TDM is known to be the best for moderate interference coefficients. For weak interference, TDM can achieve acceptable performance since the interference is low. In practice, a situation with strong interference can be made unlikely to occur with appropriate spectrum allocation. To make a trade-off between the performance and the complexity, we adopt TDM-based power control.

The vertices of the feasible power region are chosen as candidate power strategies. Let $N_{k}^{f}$ be the number of links that use spectrum $k$ on the path of flow $f . N_{k}^{f}$-dimensional feasible regions are bounded by both node peak transmit power and interference temperature constraints. In the information-theoretic TDM, only one link can transmit data in a time slot. By contrast, our proposed TDM can allow multiple links to transmit data simultaneously. We can thus achieve better performance than previously known analyses.

There are many vertices of the feasible power region, denoted as $p_{l v}$ for link $l$ at vertex $v$, whose corresponding throughput is $R_{l, v}$. The expected throughput of link $l$ is

$$
\mathbb{E}\left[R_{l}\right]=\sum_{v \in \mathcal{V}_{k}} \omega_{k(l), v} R_{l, v} P_{l}^{s u c}\left(\omega_{k, v}\right),
$$

where $\mathcal{V}_{k}$ is the set of all possible transmission combinations, $\omega_{k, v}$ is the time portion of the power strategy at vertex $v$ on spectrum $k$, and $P_{l}^{s u c}\left(\omega_{k, v}\right)$ is the successful transmission probability due to interflow transmission conflicts, which will be presented further in Section 5.1.

The problem is transformed from maximization of flow $f^{\prime}$ s end-to-end throughput to several subproblems of maximizing the minimum link throughput on each spectrum. The subproblem for spectrum $k$ can be stated as

$$
\begin{gathered}
\max \min _{l \in \mathcal{L}(f), k(l)=k}\left(\sum_{v \in \mathcal{V}_{k}} \omega_{k, v} R_{l, v} P_{l}^{s u c}\left(\omega_{k, v}\right)\right), \\
\text { s.t. } \sum_{v \in \mathcal{V}_{k}} \omega_{k, v}=1 .
\end{gathered}
$$

To achieve the optimality, data should be transmitted over all links with the same throughput, i.e., all $\mathbb{E}\left[R_{L}\right] \mathrm{s}$ are identical. Equation (11) can be transformed as

$$
\begin{gathered}
\sum_{v \in \mathcal{V}_{k}} \omega_{k, v} R_{i, v} P_{l}^{s u c}\left(\omega_{k, v}\right)=\sum_{v \in \mathcal{V}_{k}} \omega_{k, v} R_{j, v} P_{l}^{s u c}\left(\omega_{k, v}\right), \\
\forall i, j \in \mathcal{L}(f), k(i)=k, k(j)=k, i \neq j .
\end{gathered}
$$

So, we can solve (13) and (14) for $\omega_{k, v}$.

The number of candidate power vectors is always larger than that of links on the same spectrum, meaning that there are more undetermined parameters than the number of equations. Thus, the equations cannot be solved directly.

The optimal end-to-end throughput can be achieved in two cases. The first case is a linear combination of multiple power strategies. From a geometrical perspective, in the $N_{k}^{f}$-dimensional throughput space, the optimal end-to-end throughput is achieved at the maximum intersection point of the line that the throughputs of all links are the same, and the throughput polyhedron achieved by the power strategies at the vertices of the feasible power region. It must be the intersection point of the line and some $N_{k}^{f}$-dimensional hyperplane. This point can be constructed by a linear combination of at most $N_{k}^{f}$ vertices because it is on a hyperplane determined by $N_{k}^{f}$ vertices. In the second case, there exists a dominant power strategy that achieves larger throughputs of all links than those achieved by other strategies. The optimal solution adopts the dominant strategy.

The optimal power-control strategy, therefore, consists of at most $N_{k}^{f}$ strategies according to some time portion $\omega_{k, v}$. We can choose $N_{k}^{f}$ power vectors from the candidate power vectors and solve them using (13). After enumerating all the combinations for selecting power vectors, the maximum solution is chosen as the end-to-end throughput achieved on the SSR.

\subsection{Reduction of Computational Complexity}

The computational complexity of the power-control scheme increases exponentially with the number of links. Fortunately, the problem has already been decomposed into subproblems, one for each spectrum, so the number of links on a spectrum is usually not so large, lowering the complexity.

There are two other considerations in reducing the computational complexity. Omni-directional antennas are chosen for our study, and hence, it is inefficient if the node transmits and receives data on the same channel at the same time because of self-interference. Let $N^{f}$ be the number of links that flow $f$ runs through. The number of possible candidate SSRs for flow $f$ is $K(K-1)^{\left(N^{f}-1\right)}$, so the ratio of the reduced complexity is $\left(\frac{K-1}{K}\right)^{\left(N^{f}-1\right)}$ times more than the original one. If the geographic location information is available, then the node would not forward the data to any neighbor farther away from the destination [28]. To reduce the complexity, these inefficient cases are not 

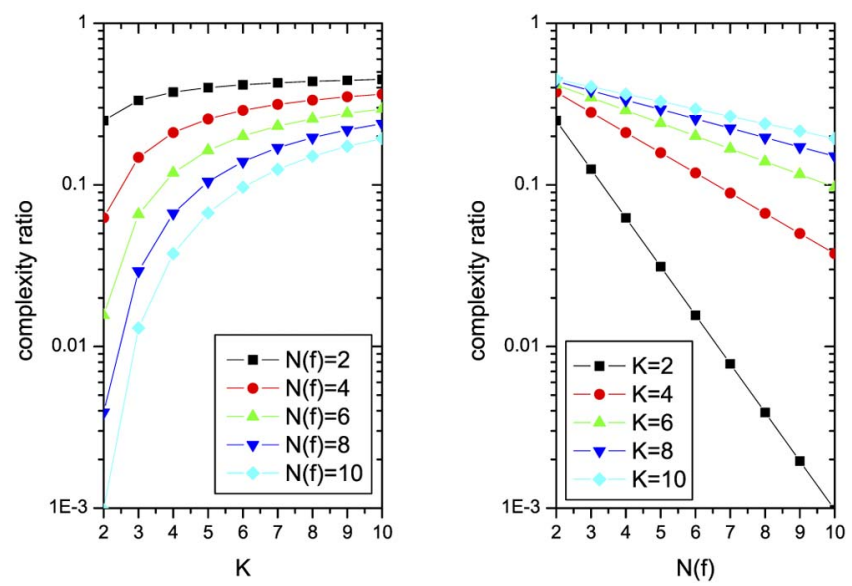

Fig. 3. The ratio of reduced complexity to original complexity with different numbers of channels and links.

considered in the algorithm. Fig. 3 shows the ratio of the reduced complexity to the original complexity. According to the figure, these considerations can reduce complexity further when the number of channels gets smaller or the number of links becomes larger.

\section{Cooperation among Multiple Flows}

For the multiflow case, the interflow interference and the cumulative interference to PUs need to be considered for the selection of an SSR and a power-control strategy.

\subsection{Cooperation on Interflow Interference}

In order to control the interflow interference, we use the RTS/CTS scheme. When a node is receiving packets, its neighbors are not allowed to transmit. This causes the endto-end throughput of the related flows to degrade, which needs to be considered in estimating the end-to-end throughput for SSRs.

To estimate the effect of collision among multiple flows, the nodes obtain the transmission probability information from their neighbors and calculate the probability of successful transmission. Since all flows are assumed to have same priority, each link has the same probability to use the spectrum if a collision occurs. For example, link A wants to transmit data with probability $\omega$, and its neighbor link transmits data with probability $\omega_{1}$. Then, the actual probability that A can transmit successfully is

$$
P^{s u c}\left(\omega, \omega_{1}\right)=1-\frac{1}{2} \omega_{1}
$$

In general, the successful transmission probability can be computed when there are $N$ neighbor links of A as

$$
\begin{aligned}
P^{s u c}(\omega)= & 1-\frac{1}{2} \sum_{1 \leq i \leq N} \omega_{i}+\frac{1}{3} \sum_{1 \leq i, j \leq N, i \neq j} \omega_{i} \omega_{j} \\
& +\cdots+(-1)^{N} \frac{1}{N+1} \omega_{1} \cdots \omega_{N} .
\end{aligned}
$$

The expression for the successful transmission probability (16) is proved to be correct via mathematical induction in Appendix A.
To calculate E2E throughput, we need to consider the successful probability. Considering the successful transmission probabilities, the power-control problem (12) in Section 4 can be solved by adjusting $\omega_{k, v}$ and $P_{l}^{s u c}\left(\omega_{k, v}\right)$ iteratively.

This way, the nodes can estimate the effect of interflow interference by using only local information from their neighbors. If this cooperation is not deployed, the nodes may estimate the interflow interference only according to the a priori known node density.

\subsection{Cooperation to Meet Cumulative Interference Temperature Constraints}

A straightforward way of meeting the interference temperature constraints is to keep the allowable interference temperatures of each flow under $1 /|\mathcal{F}|$ of the total interference temperature constraints [29]. Recall that $|\mathcal{F}|$ is the number of flows. This conservative method can limit the interference, but will result in poor spectrum utilization, as a flow may be considered to cause the rise of interference temperature even when all the nodes on the flow's path are far away from the PUs.

To get a distributed solution, we adopt decomposition based on the Lagrangian duality. Using the Lagrangian multiplier, we can rewrite the objective as

$$
\min _{\beta} D
$$

where $\beta$ is the vector of Lagrangian multipliers composed by $\beta_{m k}$ for primary user $m$ and spectrum $k$. The dual function is

$$
\begin{aligned}
D & =\max \sum_{f \in \mathcal{F}} U^{f}-\sum_{m \in \mathcal{M}} \sum_{k \in \mathcal{K}} \beta_{m k}\left(\sum_{f \in \mathcal{F}} Q_{m k}^{f}\right) \\
& =\sum_{f \in \mathcal{F}}\left(\max U^{f}-\sum_{m \in \mathcal{M}} \sum_{k \in \mathcal{K}} \beta_{m k} Q_{m k}^{f}\right) .
\end{aligned}
$$

In this method, the binding interference temperature constraint is decomposed for each flow. In order to share the allowable interference temperature among flows and improve spectrum utilization, each flow optimizes the following function:

$$
D^{f}=\max U^{f}-\sum_{m \in \mathcal{M}} \sum_{k \in \mathcal{K}} \beta_{m k} Q_{m k}^{f} .
$$

By the Lagrangian multiplier method, the maximum $\sum_{f \in \mathcal{F}} U_{f}$ is proved to be achievable by maximizing $D^{f}$ for each flow as in [30].

Note that the interference temperature information at the primary receivers is necessary for adjustment of $\beta_{m k}$. In this way, the SUs can determine $\beta_{m k}$ according to the information collected locally from the primary receivers nearby to guarantee that

$$
\sum_{f \in \mathcal{F}} Q_{m k}^{f} \leq Q_{m k}^{\max }
$$

If PUs cannot cooperate to report this required information to SUs, we need a controller that determines the values of $\beta_{m k}$ to keep the interference temperature below the upper bounds $Q_{m k}^{f}$. The controller collects the current interference 


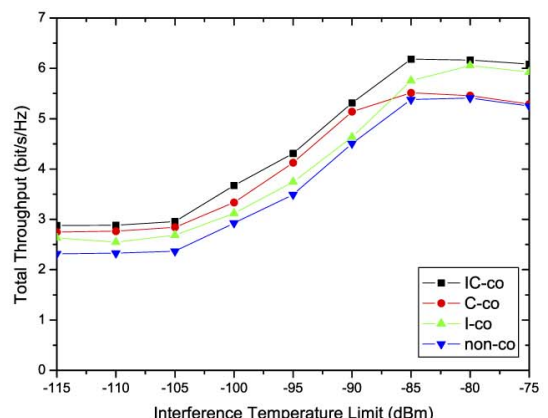

(a)

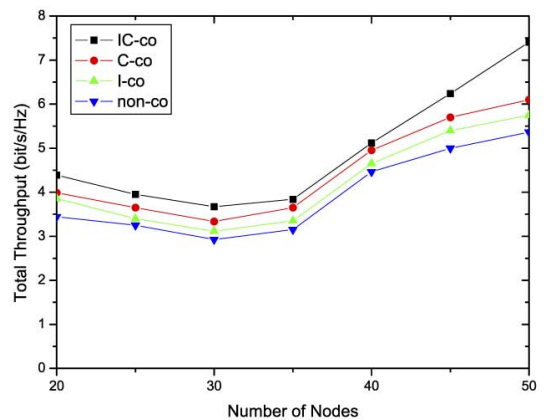

(b)

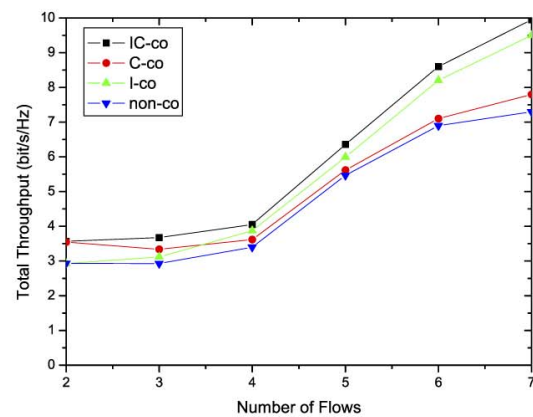

(c)

Fig. 4. Performance of the proposed algorithm with and without cooperation. (a) 30 nodes, 3 flows. (b) $-100 \mathrm{dBm}$ interference limit, 3 flows. (c) $-100 \mathrm{dBm}$ interference limit, 30 nodes.

temperature information from the primary receivers or sensors thereof. Using this information, it can adjust $\beta_{m k}$ and announce the updated value to all of the flows. It is not necessary to have a whole-network-range controller that collects the related information from the whole network. Since a node causes little interference to the nodes far away from it, the controllers only need the local information inside the interference range, which pushes the proposed scheme to be more realistic.

\subsection{Cooperation Gain}

The noncooperative scheme is considered as the baseline for comparison. Corresponding to the interflow cooperation scheme, the noncooperative scheme is just the one without considering collision between multiple flows when calculating the E2E throughput for each SSR. For the cumulative interference temperature constraints, the noncooperative scheme keeps the interference temperature of each flow below $1 /|\mathcal{F}|$ of the interference temperature constraints since there are $|\mathcal{F}|$ flows in the network. We evaluate the performance of four schemes: cooperation for both interflow interference and cumulative interference temperature constraints (IC-CO), cooperation only for interflow interference (I-Co), cooperation only for cumulative interference temperature constraints ( $\mathrm{C}-\mathrm{CO})$, and noncooperative schemes (non-co). The simulation configuration is set as in Section 7.2. We evaluate and compare the performances of the above four schemes.

Fig. 4a shows the improvement made by the proposed cooperation schemes. In this figure, with large interference temperature limits (i.e., more than $-85 \mathrm{dBm}$ in our case), the performance difference between IC-co and I-co is not noticeable; the same was also observed between C-co and non-co. This is because the cooperation on the cumulative interference temperature is unnecessary when the interference temperature is too large to restrict the transmit power.

Fig. $4 \mathrm{~b}$ shows that the proposed scheme makes significant improvements in this case. When the number of nodes is small, some flows cannot find routes to their destinations. However, for those flows that can reach their destinations, their throughput is high as a result of small interferences. When the number of nodes gets larger (e.g., 30 nodes), almost all of flows can find routes to their destinations, but only a few candidate SSRs can be found and large interferences exist between flows because of large hop counts. Note that our proposed cooperation scheme cannot eliminate the interflow interference, but takes the interference into account for selection of the SSR and the transmit power. This is why the performance of the 20-node case is better than that of the 30 -node case. When there are many nodes in the network, many routes can be found between them, and there is less interflow interference due to densely populated nodes. One can also see that for the 50-node case, the throughput of IC-co still increases as the number of nodes increases, while the other schemes are almost saturated.

In Fig. 4c, when there are only two flows, the improvement with cooperation for interflow interference is small. The difference between IC-co and C-co schemes gets larger as the number of flows increases, so does the performance difference between I-co and non-co. With a large number of flows, the cooperation for interflow interference can make significant effect. The cooperation for the cumulative interference temperature can also make a little performance improvement, but the difference for a different number of flows is not obvious from the simulation results.

All the simulation results show the improvement of the proposed cooperation schemes, which can achieve 10-40 percent more throughput than the noncooperation scheme.

\section{Optimal SSR SWITCH-WaIT TIME}

When the state of some spectrum has changed for some predefined time, both route and spectrum need to be switched, triggering the SSR selection procedures again. The optimal switch-wait time should be calculated for each spectrum. For simplicity of expression, the spectrum index $k$ is omitted in this section. We now present a method for determining the optimal wait time $T_{S}^{O N}$ and $T_{S}^{O F F}$ by making a trade-off between spectrum efficiency and switch overhead.

\subsection{Expected Throughput with Consideration of Switch Overhead}

Our objective is to find the optimal switch-wait times $T_{S}^{O N}$ and $T_{S}^{O F F}$ to maximize the total throughput over a long time period. Since ON and OFF states alternate, we consider the expected throughput of one $\mathrm{ON}$ period and one OFF period. The objective becomes maximization of the expected throughput in a single ON-OFF period, instead of the average throughput over a long time period. The expected 


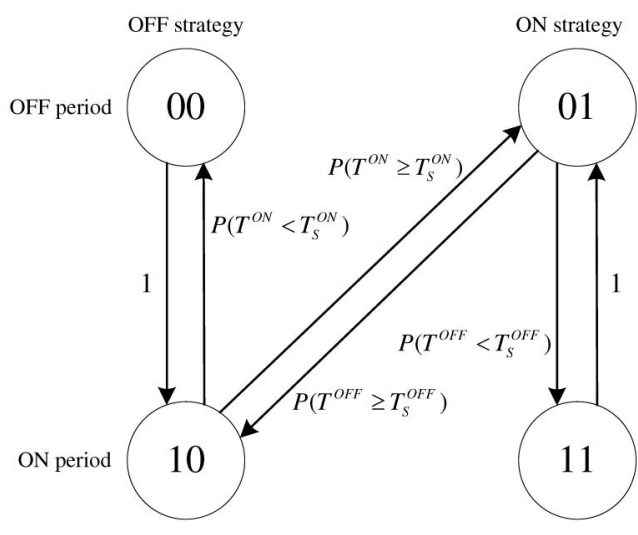

Fig. 5. The state-transition diagram.

throughput during an ON period and an OFF period can be calculated as

$$
P_{00} R_{00}+P_{01} R_{01}+P_{11} R_{11}+P_{10} R_{10}
$$

where $P_{00}$ and $P_{01}$ are the probabilities that the strategy is designed for OFF and ON states, respectively, at the beginning of an OFF state, $R_{00}$ and $R_{01}$ are the expected throughput during an OFF period beginning with a strategy designed for OFF and ON period, respectively. $P_{11}, P_{10}, R_{11}$, and $R_{10}$ are defined similarly for the ON period.

The probabilities of initial strategies can be calculated by using state-transition probabilities. Fig. 5 shows the state-transition diagram. For the beginning of an OFF state, the probabilities of strategies can be calculated by using the probabilities of all possible transitions from the beginning of the last OFF state. For steady-state transition, the probabilities of beginning strategies in each OFF state should be same. For 00 state, there are two cases, 00 (last OFF state) $\rightarrow 10$ (ON state) $\rightarrow 00$ (OFF state) and 01 (last OFF state) $\rightarrow 10$ (ON state) $\rightarrow 00$ (OFF state). It can be obtained as

$$
\begin{aligned}
P_{00}= & P_{00} P\left(T^{O N}<T_{S}^{O N}\right) \\
& +P_{01} P\left(T^{O F F} \geq T_{S}^{O F F}\right) P\left(T^{O N}<T_{S}^{O N}\right) .
\end{aligned}
$$

Similarly, the equation for 01 state can be obtained as

$$
\begin{aligned}
P_{01}= & P_{00} P\left(T^{O N} \geq T_{S}^{O N}\right)+P_{01} P\left(T^{O F F}<T_{S}^{O F F}\right) \\
& +P_{01} P\left(T^{O F F} \geq T_{S}^{O F F}\right) P\left(T^{O N} \geq T_{S}^{O N}\right) .
\end{aligned}
$$

From (22) and (23), we get

$$
\frac{P_{00}}{P_{01}}=\frac{P\left(T^{O F F} \geq T_{S}^{O F F}\right) P\left(T^{O N}<T_{S}^{O N}\right)}{P\left(T^{O N} \geq T_{S}^{O N}\right)} .
$$
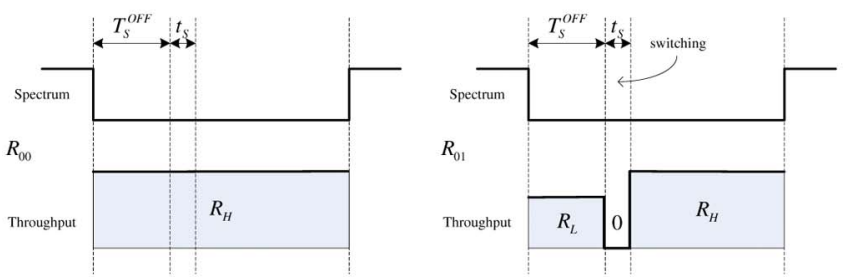

Since $P_{00}+P_{01}=1$,

$$
\begin{gathered}
P_{00}=\frac{P\left(T^{O F F} \geq T_{S}^{O F F}\right) P\left(T^{O N}<T_{S}^{O N}\right)}{P\left(T^{O F F} \geq T_{S}^{O F F}\right) P\left(T^{O N}<T_{S}^{O N}\right)+P\left(T^{O N} \geq T_{S}^{O N}\right)}, \\
P_{01}=\frac{P\left(T^{O N} \geq T_{S}^{O N}\right)}{P\left(T^{O F F} \geq T_{S}^{O F F}\right) P\left(T^{O N}<T_{S}^{O F F}\right)+P\left(T^{O N} \geq T_{S}^{O N}\right)} .
\end{gathered}
$$

This way, the steady-state probabilities of the beginning strategies are obtained for OFF state. For ON state, $P_{11}$ and $P_{10}$ can also be obtained similarly as

$P_{11}=\frac{P\left(T^{O N} \geq T_{S}^{O N}\right) P\left(T^{O F F}<T_{S}^{O F F}\right)}{P\left(T^{O N} \geq T_{S}^{O N}\right) P\left(T^{O F F}<T_{S}^{O F F}\right)+P\left(T^{O F F} \geq T_{S}^{O F F}\right)}$,

$P_{10}=\frac{P\left(T^{O F F} \geq T_{S}^{O F F}\right)}{P\left(T^{O N} \geq T_{S}^{O N}\right) P\left(T^{O F F}<T_{S}^{O F F}\right)+P\left(T^{O F F} \geq T_{S}^{O F F}\right)}$.

For calculation of the expected throughput with the beginning strategies, if the beginning strategy is consistent with the state, the SU will retain the beginning strategy. The strategies during the waiting time are different for $\mathrm{ON}$ and OFF states. The SUs do not transmit at all to protect PUs for the $\mathrm{OFF} \rightarrow \mathrm{ON}$ case, while the SUs continue to transmit using the beginning strategy for the $\mathrm{ON} \rightarrow \mathrm{OFF}$ case as shown in Fig. 6. $R_{H}$ and $R_{L}$ denote the achieved throughput of the strategies determined with the spectrum in OFF and ON periods, respectively. The expected throughput for each case can be calculated as

$$
\begin{aligned}
R_{00}= & R_{H} E\left(T^{O F F}\right) \\
R_{01}= & \int_{0}^{T_{S}^{O F F}}\left(R_{L} t\right) f^{O F F}(t) d t+\int_{T_{S}^{O F F}}^{T_{S}^{O F F}+t_{S}}\left(R_{L} T_{S}^{O F F}\right) f^{O F F}(t) d t \\
& +\int_{T_{S}^{O F F}+t_{S}}^{\infty}\left(R_{L} T_{S}^{O F F}+R_{H}\left(t-T_{S}^{O F F}-t_{S}\right)\right) f^{O F F}(t) d t,
\end{aligned}
$$

$R_{11}=R_{L} E\left(T^{O N}\right)$,

$R_{10}=\int_{T_{S}^{O N}+t_{s}}^{\infty}\left(R_{L}\left(t-T_{S}^{O N}-t_{S}\right)\right) f^{O N}(t) d t$.

We can find the optimal $T_{S}^{O N}$ and $T_{S}^{O F F}$ that maximize the throughput described as in (21). The ratio $R_{H} / R_{L}$ can be predefined with a priori knowledge, or adjusted dynamically by learning from the recently achieved performance.
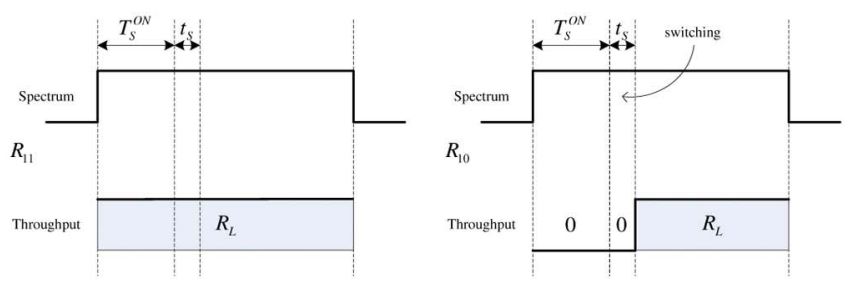

Fig. 6. Illustration of $R_{00}, R_{01}, R_{11}$, and $R_{10}$. 


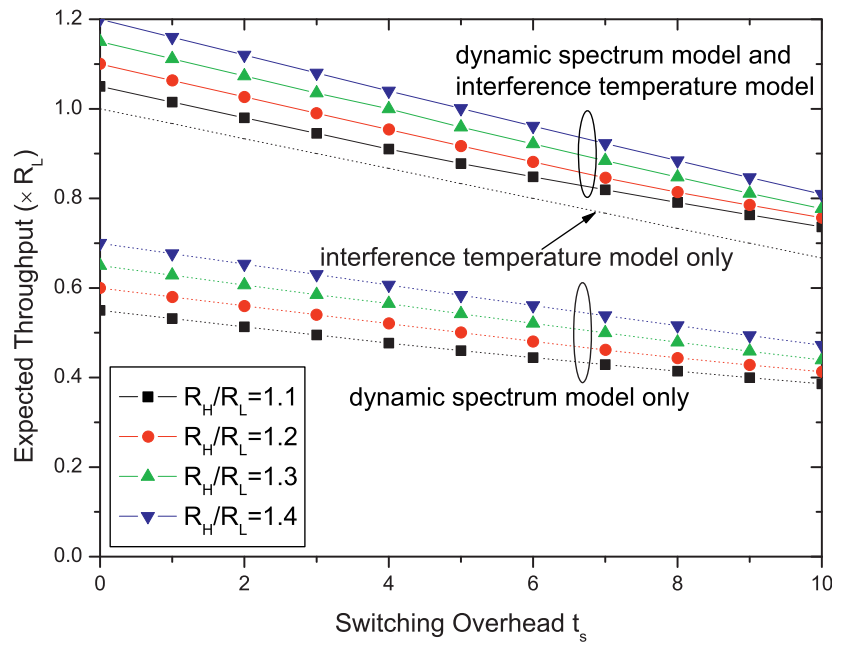

Fig. 7. Expected throughput according to different switching overheads and $R_{H} / R_{L}$.

\subsection{Optimal Waiting Time for Exponential and Erlang Distributions}

To give an insight on the optimal switch-wait time, we consider the performance for exponential and Erlangdistributed ON/OFF processes. Note that the durations of $\mathrm{ON}$ and OFF periods do not have to follow these distributions; we only use these two distributions as examples for an illustrative purpose.

In case of an exponentially distributed process due to its memoryless property, the expected remaining time would not change after waiting some time, and hence, the optimal solution is either adjust the strategy if the primary activity changes or not switch at all. We compare the above two situations. When switching from ON to OFF strategy at the beginning of OFF state, the achieved throughput is $\left(\mathbb{E}\left[T^{O F F}\right]-t_{s}\right) R_{H}$. When switching from OFF to ON strategy at the beginning of ON state, the achieved throughput is $\left(\mathbb{E}\left[T^{O N}\right]-t_{s}\right) R_{L}$. Without switching SSR, the corresponding throughput for OFF and ON states are $R_{H}$ and 0, respectively, if OFF strategy, or always $R_{L}$, if ON strategy. Therefore, the strategy should be changed if the primary activity changes as long as the following inequality holds:

$$
\begin{aligned}
& \left(\mathbb{E}\left[T^{O F F}\right]-t_{s}\right) R_{H}+\left(\mathbb{E}\left[T^{O N}\right]-t_{s}\right) R_{L} \\
& \quad>\max \left\{\mathbb{E}\left[T^{O F F}\right] R_{H},\left(\mathbb{E}\left[T^{O F F}\right]+\mathbb{E}\left[T^{O N}\right]\right) R_{L}\right\} .
\end{aligned}
$$

Otherwise, it should continue using one of the two strategies. We compare the expected throughput for ON and OFF strategies-which are $R_{L}$ and $R_{H} \mathbb{E}\left[T^{O F F}\right] /$ $\left(\mathbb{E}\left[T^{O F F}\right]+\mathbb{E}\left[T^{O N}\right]\right)$ - and choose the better of the two.

Considering an $X$-order Erlang-distributed ON/OFF process, we get

$$
\begin{gathered}
P\left(T^{O N} \geq T_{S}^{O N}\right)=\sum_{n=0}^{X-1} e^{-\lambda^{O N} T_{S}^{O N}}\left(\lambda^{O N} T_{S}^{O N}\right)^{n} / n !, \\
P\left(T^{O N}<T_{S}^{O N}\right)=1-\sum_{n=0}^{X-1} e^{-\lambda^{O N}} T_{S}^{O N}\left(\lambda^{O N} T_{S}^{O N}\right)^{n} / n !,
\end{gathered}
$$

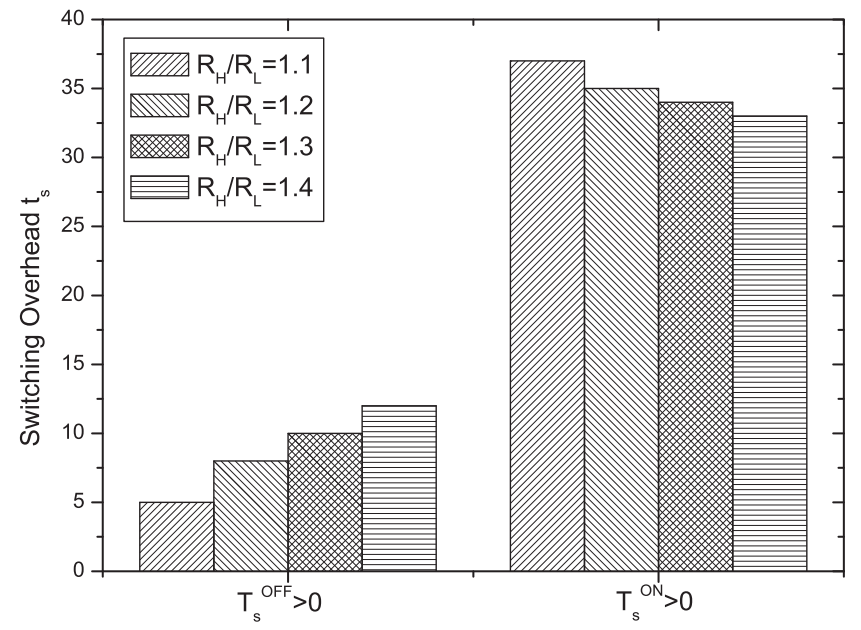

Fig. 8. Minimum switching overhead if the switch-wait time is larger than 0 .

$$
P\left(T^{O F F} \geq T_{S}^{O F F}\right)=\sum_{n=0}^{X-1} e^{-\lambda^{O F F} T_{S}^{O F F}}\left(\lambda^{O F F} T_{S}^{O F F}\right)^{n} / n !,
$$

$$
P\left(T^{O F F}<T_{S}^{O F F}\right)=1-\sum_{n=0}^{X-1} e^{-\lambda^{O F F} T_{S}^{O F F}}\left(\lambda^{O F F} T_{S}^{O F F}\right)^{n} / n !
$$

The objective function for the Erlang-distributed process can be obtained by substituting the above equations into (21). Because the probability density functions of the spectrum activities are known, the optimal waiting time can be obtained by maximizing the objective function.

Fig. 7 shows the expected throughput for a three-order Erlang-distribution process in which $\lambda^{O N}=\lambda^{O F F}=30 \mathrm{~s}$. The performance with different switching overheads and switch gain ratios $R_{H} / R_{L}$ is compared. From this figure, the expected throughputs decrease proportionally as the switching overhead increases. If the spectrum opportunities are utilized only according to the dynamic spectrum model, the performance is shown as the dash lines. Without considering the interference temperature model, when a PU is transmitting, the nearby SUs are not allowed to transmit, which causes the loss of spectrum utilization. On the contrary, if the spectrum is utilized only according to the interference temperature limit, all the users transmit as the case that the PUs are active, so the achieved throughput is $R_{L}$. This simulation results show the advantage of utilizing both models to exploit the spectrum opportunities in this paper.

With a large enough switch overhead, the waiting time should be nonzero. Fig. 8 shows the minimum switching overhead if the switching waiting time is larger than 0 . The minimum switching overhead for nonzero $T_{S}^{O F F}$ is found to be larger for larger $R_{H} / R_{L}$. In contrast, for nonzero $T_{S}^{O N}$, the smaller $R_{H} / R_{L}$, the larger minimum overhead, because the ratio of the change from 0 to $R_{L}$ to the change from $R_{L}$ to $R_{H}$ with large $R_{H} / R_{L}$ is relatively small compared to it in the case with small $R_{H} / R_{L}$.

\section{Performance Evaluation}

\subsection{Convergence}

With the estimated successful transmission probability, the interaction between the TDM-based power control and the 


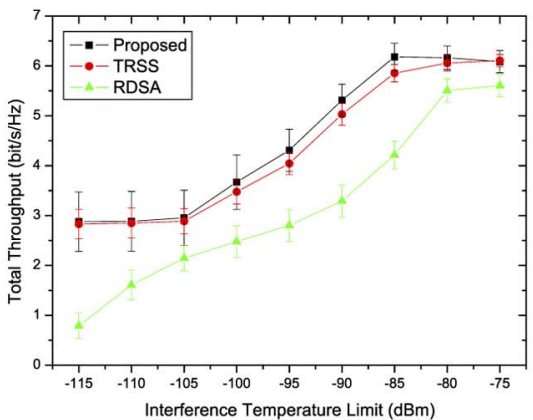

(a)

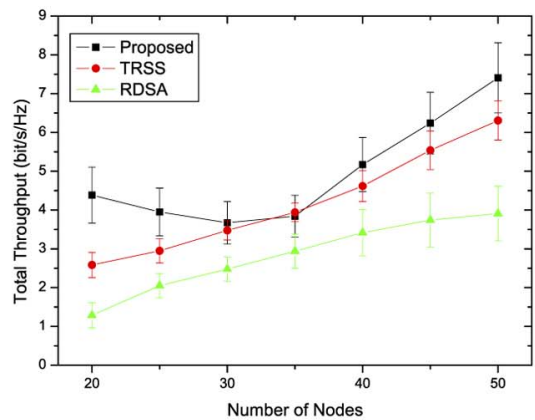

(b)

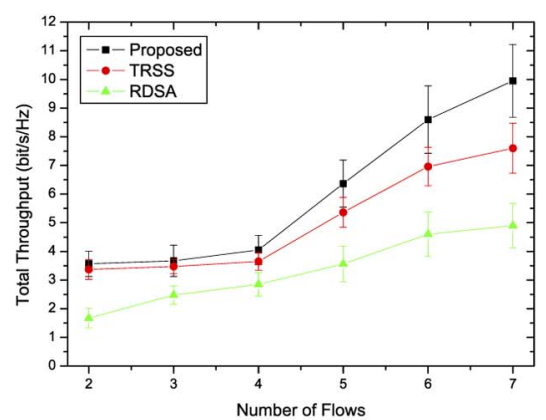

(c)

Fig. 9. Total throughput of the proposed algorithm compared with RDSA and TRSS algorithms. (a) 30 nodes, 3 flows. (b) $-100 \mathrm{dBm}$ interference limit, 3 flows. (c) $-100 \mathrm{dBm}$ interference limit, 30 nodes.

cooperation between flows takes multiple iterations to converge. Based on the definition of the standard function [31], the convergence of our proposed iterative algorithm is proved in two steps. First, $I(\omega)$ formulated from our joint spectrum allocation and power-control problem satisfies the three conditions of standard functions. Second, if $I(\omega)$ is a standard function, the iterative algorithm converges. Here, we provide the first step of the proof, but omit the details of the second part since the proof of the second part is the same as that in [31].

Consider a set of links which is within the interference range of each other. Let $\omega$ and $\mathbf{P}^{s u c}(\omega)$ denote the vectors of their transmission time portions and the transmission success probabilities, respectively. The probability $\mathbf{P}^{\text {suc }}(\omega)$ is updated according to (16), while the allocated transmission time portion is adjusted as

$$
\omega \geq \mathbf{I}(\omega) .
$$

In order to guarantee the efficient transmission time $\eta$, the update function of $\omega$ in the proposed iterative algorithm is presented as

$$
\mathbf{I}_{l}(\boldsymbol{\omega})=\frac{\eta_{l}}{P_{l}^{s u c}(\omega)} .
$$

In [31], the iterative algorithm can converge to a unique fixed point if the update function is a standard function. The definition of the standard function has three conditions for all $\omega>0$ :

- Positivity: $\mathbf{I}(\omega)>0$.

- Monotonicity: If $\omega \geq \omega^{\prime}$, then $\mathbf{I}(\omega) \geq \mathbf{I}\left(\omega^{\prime}\right)$.

- Scalability: For all $\alpha>1, \alpha \mathbf{I}(\omega)>\mathbf{I}(\alpha \omega)$.

The positivity and the monotonicity of the update function are satisfied according to the expression of the successful transmission probability (16). For the scalability, the condition is met if and only if $P_{l}^{s u c}(\omega)$ satisfies

$$
P_{l}^{s u c}(\alpha \omega)>\frac{P_{l}^{s u c}(\omega)}{\alpha} .
$$

The detailed proof of this scalability is provided in Appendix B. Since the update function of the proposed iterative algorithm satisfies the conditions, the algorithm converges.

The Lagrangian multipliers are relatively constant during the execution time of the iterative algorithm of the
TDM-based power control and the cooperation for interflow interference. If the adjusting step size $\delta$ satisfies the following expressions, the algorithm can eventually converge to a unique point [32].

$$
\delta^{n} \geq 0, \quad \lim _{n \rightarrow \infty} \delta^{n}=0, \quad \sum_{n=0}^{\infty} \delta^{n}=\infty, \quad \sum_{n=0}^{\infty}\left(\delta^{n}\right)^{2}<\infty .
$$

\subsection{Performance Comparison}

The performance of the proposed algorithms is evaluated using dynamic system-level simulation with OPNET. Primary and secondary users/devices/nodes are uniformly placed in a $100 \mathrm{~m} \times 100 \mathrm{~m}$ area. The path gain between nodes is modeled as $P L=P L_{0} \cdot D / D_{0}$, where $D$ is distance. $P L_{0}$ and $D_{0}$ are set to $90 \mathrm{~dB}$ and $1 \mathrm{~m}$, respectively. The peak transmit power of secondary nodes is set to $20 \mathrm{dBm}$. There are two primary nodes whose interference caused by the secondary nodes should not exceed the interference temperature limit at the primary nodes. The interference temperature constraints are assumed identical for the two primary nodes. The spectrum activity is modeled as an ON/OFF process in which the lengths of ON and OFF periods are assumed to be exponentially distributed. The average durations of both $\mathrm{ON}$ and OFF periods are $30 \mathrm{~s}$ for these spectra. To compute the average performance, 50 different topologies are considered, and the simulation ran for 20 minutes for each of them. Selection of these numbers is arbitrary, but it does not change the conclusions we draw from the simulation. The 95 percent confidence interval of simulation results is shown using error bars.

For performance comparison, we consider two existing schemes, RDSA [8] and TRSS [20]. RDSA maximizes the E2E throughput by link scheduling with cochannel interference, and TRSS considers link scheduling for spectrum sharing together with a binary interference model. The simulation results in Fig. 9 demonstrate the performance improvement of the proposed algorithm which considers power control together with other aspects of resource allocation.

Compared with TRSS, in Fig. 9a, the performance improvement is not obvious when the interference temperature limit is large, because the power control is not so important if the transmit power is not restricted. Fig. 9b shows the proposed scheme to achieve better performance when the number of secondary nodes is small or large 
enough. When the number of secondary nodes is moderate (e.g., 30 nodes), the performances of TRSS and the proposed algorithm are similar because of serious interflow conflicts, as same as it shown in Fig. 4. Fig. 9c shows the performance improvement to increase as the number of flows increases. The confidence interval of the proposed algorithm is larger than TRSS because the effect of power control in the proposed algorithm makes a significant performance gain in certain (not all) topologies, causing the large confidence interval.

From the simulation results, one can see that the performance of RDSA is much worse than that of the proposed scheme in CRNs with interference temperature limits. The maximum power is used, causing too large interference to primary users. Therefore, a number of links near PUs are unavailable. Especially, in Fig. 9a, when the interference temperature limit is tight (e.g., $-115 \mathrm{dBm}$ ), almost all the links are disconnected and only a few flows can be transmitted from sources to destinations successfully, so the average total throughput is low. In such a case, for RDSA algorithm, the ratio of the confidence interval to the average value is large.

The confidence interval decreases with the increasing number of random topologies. Here, we pay more attention to the relative values of the proposed algorithm in various scenarios with different parameters. In most cases, as shown in Figs. $9 b$ and $9 c$, the confidence interval is larger if the corresponding average value is larger. By contrast, in Fig. 9a, when the interference temperature limit increases, the total throughput increases with the decreasing confidence interval. When the interference temperature limit is small, the random positions of primary users affect the stability of throughput performance.

\section{Conclusions AND Future Work}

In this paper, joint spectrum allocation and power control is proposed to utilize open spectrum bands in CRNs by considering both interference temperature constraints and spectrum dynamics. A TDM-based power-control strategy is adopted to achieve maximum end-to-end throughput, which is used to evaluate the performance of each candidate SSR in choosing an appropriate multihop route and its corresponding spectrum for a single flow. In multiflow networks, each flow determines its own strategy in a distributed manner. Both interflow and cumulative interference temperatures are considered in the cooperative schemes. Considering the overhead of switching routes and spectra, the optimal wait time is derived using a probabilistic approach. A balance is achieved by making a trade-off between spectrum efficiency and the overhead of switching routes and spectrum bands. Our simulation results show that the proposed cooperative schemes utilize the spectrum very efficiently.

In this paper, the spectrum bands are assumed independent of each other. The interaction between spectrum bands will be considered in our future work. The authors of [33] showed that the spectrum activities are correlated in the frequency domain. The phenomenon of power leakage also increases the interference between adjacent channels.

\section{APPENDIX A}

\section{Proof of Successful Transmission Probability}

Following the discussion in Section 5.1, in order to accurately evaluate the end-to-end throughput of candidate SSRs, the successful transmission probability is proposed to account for the conflict between multiple flows. The expression of the successful transmission probability can be proved to be correct by mathematical induction.

The successful transmission probability (16) can be rewritten as

$$
\begin{aligned}
P_{\text {suc }}\left(\omega, \omega_{1}, \ldots, \omega_{N}\right)= & 1-\phi_{1} \sum_{1 \leq i \leq N} \omega_{i} \\
& +\phi_{2} \sum_{1 \leq i, j \leq N, i \neq j} \omega_{i} \omega_{j} \\
& +\cdots+\phi_{N} \omega_{1} \cdots \omega_{N},
\end{aligned}
$$

where the coefficients are

$$
\phi_{n}=(-1)^{n} \frac{1}{n+1} .
$$

For easier understanding, we take link A with two neighbor links as an example. The probability that link A wants to transmit is $\omega$, but the actual successful transmission probability is lower than $\omega$ due to collisions. The probability that link A's transmission conflicts with one of two neighbor links is $\omega \omega_{1}$ and $\omega \omega_{2}$, respectively. In these two cases, link A can occupy the spectrum with only 50 percent of the time. To compute the successful transmission probability, $1 / 2\left(\omega \omega_{1}+\omega \omega_{2}\right)$ is subtracted from the transmission probability. However, there is a case when all three links want to transmit at the same time. The probability of such an event is $\omega \omega_{1} \omega_{2}$, and $(1-1 / 3) \omega \omega_{1} \omega_{2}$ should be subtracted from the transmission probability. This part must be subtracted $C_{2}^{1}$ times for collision between link $\mathrm{A}$ and one of its two neighbor links.

This way, the coefficient for the collision of link A and $n$ neighbor links can be calculated as

$$
\phi(n)=-\left(1-\frac{1}{n+1}\right)-\sum_{k=1}^{n-1} C_{n}^{k} \phi_{k},
$$

where

$$
C_{n}^{k}=\frac{n !}{(n-k) ! k !} .
$$

The next step is to derive the coefficients of the expression (see (43)). The first coefficient $\phi_{1}$ can be calculated by using (44):

$$
\phi_{1}=-\left(1-\frac{1}{1+1}\right)=-\frac{1}{2}=(-1)^{1} \frac{1}{1+1} .
$$

Similarly, when $n=2, \phi_{2}$ can be written as

$$
\phi_{2}=-\left(1-\frac{1}{2+1}\right)-C_{2}^{1}\left(-\frac{1}{2}\right)=\frac{1}{3}=(-1)^{2} \frac{1}{2+1} .
$$

For these basic cases, the coefficient expression (43) holds. Suppose (43) holds for all the elements from $\phi_{1}$ to $\phi_{n}$. For $\phi_{n+1}$, 


$$
\begin{aligned}
\phi_{n+1} & =-\left(1-\frac{1}{n+2}\right)-\sum_{k=1}^{n} C_{n+1}^{k} \phi_{k} \\
& =-\frac{n+1}{n+2}-\sum_{k=1}^{n}\left(C_{n+1}^{k}(-1)^{k} \frac{1}{k+1}\right) .
\end{aligned}
$$

Because of

$$
\frac{1}{k+1} C_{n+1}^{k}=\frac{1}{n+2} C_{n+2}^{k+1}
$$

$\phi_{n+1}$ can be expressed as

$$
\phi_{n+1}=-\frac{n+1}{n+2}-\frac{1}{n+2} \sum_{k=1}^{n}\left(C_{n+2}^{k+1}(-1)^{k}\right) .
$$

Substituting $k$ for $k+1$,

$$
\begin{aligned}
\phi_{n+1}= & -\frac{n+1}{n+2}+\frac{1}{n+2} \sum_{k=2}^{n+1}\left(C_{n+2}^{k}(-1)^{k}\right) \\
= & -\frac{n+1}{n+2}+\frac{1}{n+2} \cdot\left(\sum_{k=0}^{n+2} C_{n+2}^{k}(-1)^{k} 1^{n+2-k}\right. \\
& \left.-1+(n+2)-(-1)^{n+2}\right) \\
= & -(-1)^{n+2} \frac{1}{n+2} \\
= & (-1)^{n+1} \frac{1}{(n+1)+1} .
\end{aligned}
$$

So, (43) holds for $\phi_{n+1}$. Therefore, by mathematical induction, for all $n \geq 1$, the coefficients can be described as (43). Thus, the expression of the successful transmission probability follows.

\section{APPENDIX B}

\section{SCALABILITY OF the Update Function}

To verify the scalability, we need to prove $P_{l}^{s u c}(\alpha \omega)>$ $P_{l}^{\text {suc }}(\omega) / \alpha$. When $\alpha>1$,

$$
\begin{aligned}
P_{l}^{\text {suc }}(\alpha \omega)= & 1-\frac{1}{2} \alpha \sum_{1 \leq i \leq N} \omega_{i}+\frac{1}{3} \alpha^{2} \sum_{1 \leq i, j \leq N, i \neq j} \omega_{i} \omega_{j} \\
& +\cdots+(-\alpha)^{N} \frac{1}{N+1} \omega_{1} \cdots \omega_{N} .
\end{aligned}
$$

For simplicity of expression, we define $\psi_{i}$ to indicate the combinations of $i$ entries of $\omega$ and rewrite (51) as

$$
P_{l}^{s u c}(\alpha \omega)=1-\frac{1}{2} \alpha \psi_{1}+\frac{1}{3} \alpha^{2} \psi_{2}+\cdots+(-\alpha)^{N} \frac{1}{N+1} \psi_{N} .
$$

The condition of scalability is presented as

$$
\begin{gathered}
\alpha-\frac{1}{2} \alpha^{2} \psi_{1}+\frac{1}{3} \alpha^{3} \psi_{2}+\cdots-(-\alpha)^{N+1} \frac{1}{N+1} \psi_{N} \\
>1-\frac{1}{2} \psi_{1}+\frac{1}{3} \psi_{2}+\cdots+(-1)^{N} \frac{1}{N+1} \psi_{N} .
\end{gathered}
$$

The above condition can be transformed to

$$
\begin{aligned}
(\alpha-1) & -\frac{1}{2}\left(\alpha^{2}-1\right) \psi_{1}+\frac{1}{3}\left(\alpha^{3}-1\right) \psi_{2}+\cdots \\
& +(-1)^{N} \frac{1}{N+1}\left(\alpha^{N+1}-1\right) \psi_{N}>0 .
\end{aligned}
$$

Because of $\alpha-1>0$,

$$
\begin{aligned}
1 & -\frac{1}{2}(\alpha+1) \psi_{1}+\frac{1}{3}\left(\alpha^{2}+\alpha+1\right) \psi_{2}+\cdots \\
& +(-1)^{N} \frac{1}{N+1}\left(\alpha^{N}+\alpha^{N-1}+\cdots+1\right) \psi_{N}>0 .
\end{aligned}
$$

According to the relationship of sets, it can be obtained that

$$
\psi_{k}>\alpha \psi_{k+1} \text {. }
$$

Based on (56), two neighbor terms can be canceled by each other.

$$
\begin{aligned}
& \frac{1}{k+1}\left(\alpha^{k}+\alpha^{k-1}+\cdots+1\right) \psi_{k} \\
& \quad-\frac{1}{k+2}\left(\alpha^{k+1}+\alpha^{k}+\cdots+1\right) \psi_{k+1} \\
& \quad>\left(\frac{1}{(k+1)(k+2)}\left(\alpha^{k+1}+\alpha^{k}+\cdots+1\right)-\frac{1}{k+2}\right) \psi_{k+1} \\
& \quad>0 .
\end{aligned}
$$

When $N$ is an odd number, (55) is satisfied as

$$
\begin{aligned}
1- & \frac{1}{2}(\alpha+1) \psi_{1}+\frac{1}{3}\left(\alpha^{2}+\alpha+1\right) \psi_{2}+\cdots \\
& +(-1)^{N} \frac{1}{N+1}\left(\alpha^{N}+\alpha^{N-1}+\cdots+1\right) \psi_{N} \\
= & \left(1-\frac{1}{2}(\alpha+1) \psi_{1}\right)+\cdots \\
& +\left((-1)^{N-1} \frac{1}{N}\left(\alpha^{N-1}+\cdots+1\right) \psi_{N-1}\right. \\
& \left.+(-1)^{N} \frac{1}{N+1}\left(\alpha^{N}+\alpha^{N-1}+\cdots+1\right) \psi_{N}\right)
\end{aligned}
$$

$>0$

When $N$ is an even number, (55) is also satisfied as

$$
\begin{aligned}
1 & -\frac{1}{2}(\alpha+1) \psi_{1}+\frac{1}{3}\left(\alpha^{2}+\alpha+1\right) \psi_{2}+\cdots \\
& +(-1)^{N} \frac{1}{N+1}\left(\alpha^{N}+\alpha^{N-1}+\cdots+1\right) \psi_{N} \\
= & \left(1-\frac{1}{2}(\alpha+1) \psi_{1}\right)+\cdots \\
& +\left((-1)^{N-2} \frac{1}{N-1}\left(\alpha^{N-2}+\cdots+1\right) \psi_{N-2}\right. \\
& \left.+(-1)^{N-1} \frac{1}{N}\left(\alpha^{N-1}+\cdots+1\right) \psi_{N-1}\right) \\
& +(-1)^{N} \frac{1}{N+1}\left(\alpha^{N}+\alpha^{N-1}+\cdots+1\right) \psi_{N} \\
> & \frac{1}{N+1}\left(\alpha^{N}+\alpha^{N-1}+\cdots+1\right) \psi_{N}>0 .
\end{aligned}
$$


Therefore, the update function of the proposed iterative algorithm satisfies the scalability condition of the standard function.

\section{ACKNOWLEDGMENTS}

This work was supported by the National 973 Program under grant no. 2009CB320405, the National Natural Science Foundation of China under grant nos. 61001098, 60802012, 60972057, and 60972058, Fundamental Research Funds for the Central Universities under grant no. 2010QNA5011, and the Scientific Research Fund of Zhejiang Provincial Education Department under grant no. Y200909796. Wei Wang's work was partly done during his PhD study at the Beijing University of Posts and Telecommunications (BUPT) and his visit to the Real-Time Computing Laboratory (RTCL) of the University of Michigan. Kang G. Shin's work was supported by the US National Science Foundation under grants CNS-0519498 and CNS-0721529 and Intel Corporation. Wenbo Wang's work was sponsored by Nokia Corporation, the National Natural Science Foundation of China under grant nos. 60572120 and 60602058, and the National 973 Program under grant no. 2007CB310602.

\section{References}

[1] FCC, “Spectrum Policy Task Force Report," ET Docket No 02-135, Nov. 2002.

[2] J. Mitola and G. Maguire, "Cognitive Radio: Making Software Radios More Personal," IEEE Personal Comm., vol. 6, no. 4, pp. 1318, Aug. 1999.

[3] S. Haykin, "Cognitive Radio: Brain-Empowered Wireless Communications," IEEE J. Selected Areas in Comm., vol. 23, no. 2, pp. 201-220, Feb. 2005.

[4] I.F. Akyildiz, W.Y. Lee, M.C. Vuran, and S. Mohanty, "NeXt Generation/Dynamic Spectrum Access/Cognitive Radio Wireless Networks: A Survey," Computer Networks, vol. 50, no. 13, pp. 21272159, Sept. 2006.

[5] R. Cruz and A. Santhanam, "Optimal Routing, Link Scheduling and Power Control in Multi-Hop Wireless Networks," Proc. IEEE INFOCOM, pp. 702-711, Mar. 2003.

[6] Y. Xi and E. Yeh, "Distributed Algorithms for Spectrum Allocation, Power Control, Routing and Congestion Control in Wireless Networks," Proc. ACM MobiHoc, pp. 180-189, Sept. 2007.

[7] J. Tang, G. Xue, C. Chandler, and W. Zhang, "Link Scheduling with Power Control for Throughput Enhancement in Multihop Wireless Networks," IEEE Trans. Vehicular Technology, vol. 5, no. 3, pp. 733-742, May 2006.

[8] Y. Shen, Y. Zhang, and W. Wong, "Cross-Layer Link Scheduling for End-To-End Throughput Maximization in Wireless Ad Hoc Networks," Proc. IEEE Wireless Comm. and Networking Conf. (WCNC '07), pp. 282-287, Mar. 2007.

[9] Q. Wang and H. Zheng, "Route and Spectrum Selection in Dynamic Spectrum Networks," Proc. IEEE Consumer Comm. and Networking Conf. (CCNC '06), pp. 625-629, Jan. 2006.

[10] R. Pal, "Efficient Routing Algorithms for Multi-Channel Dynamic Spectrum Access Networks," Proc. IEEE Int'l Symp. New Frontiers in Dynamic Spectrum Access Networks (DySPAN '07), pp. 288-291, Apr. 2007.

[11] G. Cheng, W. Liu, Y. Li, and W. Cheng, "Joint On-Demand Routing and Spectrum Assignment in Cognitive Radio Networks," Proc. IEEE Int'l Conf. Comm. (ICC '07), pp. 6499-6503, June 2007

[12] C. Peng, H. Zheng, and B.Y. Zhao, "Utilization and Fairness in Spectrum Assignment for Opportunistic Spectrum Access," ACM Mobile Networks and Applications, May 2006.

[13] J. Tang, S. Misra, and G. Xue, "Joint Spectrum Allocation and Scheduling for Fair Spectrum Sharing in Cognitive Radio Wireless Networks," Computer Networks, vol. 52, pp. 2148-2158, Aug. 2008.
[14] W. Wang, W. Wang, Q. Lu, and T. Peng, “An Uplink Resource Allocation Scheme for OFDMA-Based Cognitive Radio Networks," Int'l J. Comm. Systems, vol. 22, no. 5, pp. 603-623, May 2009.

[15] W.Y. Lee and I.F. Akyildiz, "Joint Spectrum and Power Allocation for Inter-Cell Spectrum Sharing in Cognitive Radio Networks," Proc. IEEE Symp. New Frontiers in Dynamic Spectrum Access Networks (DySPAN '08), pp. 276-287, Oct. 2008.

[16] Y. Xing, C.N. Mathur, M.A. Haleem, R. Chandramouli, and K.P. Subbalakshmi, "Dynamic Spectrum Access with QoS and Interference Temperature Constraints," IEEE Trans. Mobile Computing, vol. 6, no. 4, pp. 423-433, Apr. 2007

[17] M. Ma and D.H.K. Tsang, "Joint Spectrum Sharing and Fair Routing in Cognitive Radio Networks," Proc. IEEE Consumer Comm. and Networking Conf. (CCNC'08), pp. 978-982, Jan. 2008.

[18] Y.T. Hou, Y. Shi, and H.D. Sherali, "Spectrum Sharing for MultiHop Networking with Cognitive Radios," IEEE J. Selected Areas in Comm., vol. 26, no. 1, pp. 146-155, Jan. 2008.

[19] Y. Shi and Y.T. Hou, "A Distributed Optimization Algorithm for Multi-Hop Cognitive Radio Network," Proc. IEEE INFOCOM, pp. 1292-1300, Apr. 2008.

[20] Z. Feng and Y. Yang, "Joint Transport, Routing and Spectrum Sharing Optimization for Wireless Networks with FrequencyAgile Radios," Proc. IEEE INFOCOM, pp. 1665-1673, Apr. 2009.

[21] A. Motamedi and A. Bahai, "MAC Protocol Design for SpectrumAgile Wireless Networks: Stochastic Control Approach," Proc. IEEE Symp. New Frontiers in Dynamic Spectrum Access Networks (DySPAN '07), pp. 448-451, Apr. 2007.

[22] S. Geirhofer, L. Tong, and B.M. Sadler, "Dynamic Spectrum Access in the Time Domain: Modeling and Exploiting White Space," IEEE Comm. Magazine, vol. 45, no. 5, pp. 66-72, May 2007.

[23] H. Kim and K.G. Shin, "Efficient Discovery of Spectrum Opportunities with MAC-Layer Sensing in Cognitive Radio Networks," IEEE Trans. Mobile Computing, vol. 7, no. 5, pp. 533545, May 2008

[24] M. Costa, "On the Gaussian Interference Channel," IEEE Trans. Information Theory, vol. 31, no. 5, pp. 607-615, Sept. 1985.

[25] I. Sason, "On Achievable Rate Regions for the Gaussian Interference Channel," IEEE Trans. Information Theory, vol. 50, no. 6, pp. 1345-1356, June 2004.

[26] Y. Yang, J. Wang, and R. Kravets, "Designing Routing Metrics for Mesh Networks," Proc. IEEE Workshop Wireless Mesh Networks (WiMesh '05), Sept. 2005

[27] A. Subramanian, M. Buddhikot, and S. Miller, "Interference Aware Routing in Multi-Radio Wireless Mesh Network," Proc. IEEE Workshop Wireless Mesh Networks (WiMesh '06), pp. 55-63, Sept. 2006

[28] B. Karp and H.T. Kung, "GPSR: Greedy Perimeter Stateless Routing for Wireless Networks," Proc. ACM MobiCom, pp. 243254, 2000.

[29] W. Wang, Y. Cui, T. Peng, and W. Wang, "Noncooperative Power Control Game with Exponential Pricing for Cognitive Radio Network," Proc. IEEE Vehicular Technology Conf. (VTC '07), pp. 3125-3129, 2007

[30] D. Tse and S. Hanly, "Multiaccess Fading Channels-Part I: Polymatroid Structure, Optimal Resource Allocation and Throughput Capacities," IEEE Trans. Information Theory, vol. 44, no. 7, pp. 2796-2815, July 1998.

[31] R.D. Yates, "A Framework of Uplink Power Control in Cellular Radio Systems," IEEE J. Selected Areas Comm., vol. 13, no. 7, pp. 1341-1347, Sept. 1995.

[32] K. Kar, S. Sarkar, and L. Tassiulas, "A Simple Rate Control Algorithm for Maximizing Total User Utility," Proc. IEEE INFOCOM, pp. 39-43, Apr. 2001.

[33] D. Chen, S. Yin, Q. Zhang, M. Liu, and S. Li, "Mining Spectrum Usage Data: A Large-Scale Spectrum Measurement Study," Proc. ACM MobiCom, pp. 13-24, Sept. 2009. 


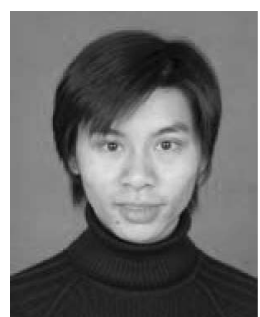

Wei Wang received the BS degree in communication engineering and the $\mathrm{PhD}$ degree in signal and information processing in 2004 and 2009, respectively, both from the Beijing University of Posts and Telecommunications (BUPT), P.R. China. Now, he is an assistant professor in the Department of Information Science and Electronic Engineering, Zhejiang University, P.R. China. From Sept. 2007 to Sept. 2008, he worked as a visiting scholar in the Real-Time Computing Lab (RTCL) at the University of Michigan, Ann Arbor. He serves as the editor for the Transactions on Emerging Telecommunication Technologies. He is the TPC cochair of CRNet 2010. His research interest mainly focuses on cognitive radio networks and radio resource management for wireless networks. He is a member of the IEEE.

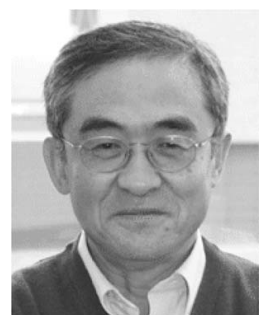

Kang G. Shin is the Kevin and Nancy O'Connor professor of computer science and the founding director of the Real-Time Computing Laboratory in the Department of Electrical Engineering and Computer Science, University of Michigan, Ann Arbor. His current research focuses on secure, QoS-sensitive networking and computing as well as on cyber-physical systems. He has supervised the completion of $65 \mathrm{PhD}$ theses, and authored/coauthored about 700 technical papers (more than 250 of which are in archival journals) and numerous book chapters. He coauthored (jointly with C.M. Krishna) the textbook RealTime Systems (McGraw Hill, 1997). He has received a number of best paper awards, including the IEEE Communications Society William R. Bennett Prize Paper Award in 2003, the Best Paper Award from IWQoS in 2003, and an Outstanding IEEE Transactions on Automatic Control Paper Award in 1987. He has also coauthored papers with his students which received Best Student Paper Awards from IEEE RTAS 1996 and the 2000 UNSENIX Technical Conference. He has also received several institutional awards, including the Research Excellence Award in 1989, the Outstanding Achievement Award in 1999, the Service Excellence Award in 2000, the Distinguished Faculty Achievement Award in 2001, the Stephen Attwood Award in 2004 from the University of Michigan, a Distinguished Alumni Award of the College of Engineering, Seoul National University in 2002, the 2003 IEEE RTC Technical Achievement Award, and the 2006 Ho-Am Prize in Engineering. He served as the general cochair for ACM MobiCom 2009; as the general chair for IEEE SECON 2008, the third ACM/USENIX MobiSys 2005, IEEE RTAS 2000, and IEEE RTSS 1987; as the program chair of IEEE RTSS 1986; and on numerous technical program committees. He also chaired the IEEE Technical Committee on Real-Time Systems during 1991-1993, was an editor of the IEEE Transactions on Parallel and Distributed Computing, and was an area editor of the International Journal of Time-Critical Computing Systems, Computer Networks, and the ACM Transactions on Embedded Systems. He is a fellow of the IEEE and the ACM.

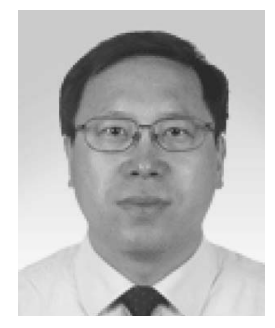

Wenbo Wang received the $\mathrm{BS}$, MS, and $\mathrm{PhD}$ degrees from the Beijing University of Posts and Telecommunications (BUPT), P.R. China, in 1986, 1989, and 1992, respectively. From 2002 to 2003 , he worked at the University of Texas, Dallas, as a visiting scholar. He is now a professor at BUPT, in charge of the Wireless Signal Processing and Network (WSPN) Lab. The main research interest of the WSPN Lab lies in key technology and theory of wireless communication, signal processing in mobile networks, and the planning and optimizing of wireless networks. He is a member of the IEEE, a council member of the China Institute of Communications, the executive director of the Beijing Institute of Communications, and deputy director of the advisory committee for the Key Laboratory of Universal Wireless Communications.

For more information on this or any other computing topic, please visit our Digital Library at www.computer.org/publications/dlib. 